TRANSACTIONS OF THE

AMERICAN MATHEMATICAL SOCIETY

Volume 351, Number 4, April 1999, Pages 1403-1422

S 0002-9947(99)02086-3

\title{
GEOMETRIC GROUPS. I
}

\author{
VALERA BERESTOVSKII, CONRAD PLAUT, AND CORNELIUS STALLMAN
}

\begin{abstract}
We define a geometry on a group to be an abelian semigroup of symmetric open sets with certain properties. Examples include well-known structures such as invariant Riemannian metrics on Lie groups, hyperbolic groups, and valuations on fields. In this paper we are mostly concerned with geometries where the semigroup is isomorphic to the positive reals, which for Lie groups come from invariant Finsler metrics. We explore various aspects of these geometric groups, including a theory of covering groups for arcwise connected groups, algebraic expressions for invariant metrics and inner metrics, construction of geometries with curvature bounded below, and finding geometrically significant curves in path homotopy classes.
\end{abstract}

\section{INTRODUCTION}

In this paper we begin a study of geometries on groups. Our definition of "geometry" is based on the observation that the metric balls centered at the identity of a (left-)invariant inner metric form a semigroup ordered by inclusion.

Definition 1.1. Let $G$ be a topological group. A geometry on $G$ consists of a basis $\left\{U_{s}\right\}_{s \in S}$ for the topology of $G$ at the identity $e$, where $(S,+, \leq)$ is a partially ordered abelian semigroup, having the following properties for all $s, t \in S$ :

G1: $U_{s} \subset U_{t}$ if and only if $s \leq t$,

G2: $\bigcap_{S} U_{s}=\{e\}$,

G3: $\bigcup_{s \in S} U_{s}=G$,

G4: $U_{s}$ is symmetric (i.e., $U_{s}^{-1}=U_{s}$ ), and

G5: $U_{s} \cdot U_{t}=U_{s+t}$.

For subsets $A, B \subset G$, by $A B$ we mean $\{x y: x \in A$ and $y \in B\}$. The requirement that $S$ be abelian will be useful for results in a later paper. Also, in the present paper $S$ is always totally ordered, but requiring this condition in Definition 1.1 seems too restrictive. The notation "+" will be used except when we are using an alternative operation on the reals or some subset (e.g. Example 1.6). We will denote simply by $\mathrm{R}^{+}$the positive reals with its usual ordering and sum operation.

Received by the editors February 14, 1997.

1991 Mathematics Subject Classification. Primary 22D05, 53C21, 53C23, 53C70.

Key words and phrases. Topological groups, Lie groups, invariant metrics, Alexandrov curvature bounded below, universal covering group.

The paper was partly written while the first author was visiting the University of Tennessee, and he wishes to acknowledge the support of the Tennessee Science Alliance and the Mathematics Department.

The second and third authors were partly supported by NSF grant DMS-9401302, and the second by a UTK Faculty Development Award.

(C)1999 American Mathematical Society 
If Definition 1.1 is satisfied, we say that $G$ is a geometric group (parameterized) over $S$. (We will sometimes say that $\left\{U_{s}\right\}$ is a geometry over $S$ when in fact $\left\{U_{s}\right\}$ is parameterized over a subsemigroup of $S$. We say that a geometry on $G$ is strong if the closures $\bar{U}_{s}$ of the members of the geometry also form a semigroup; that is $\bar{U}_{s} \cdot \bar{U}_{t}=\bar{U}_{s+t}$. Since $U_{s+t}=U_{s} U_{t} \subset \bar{U}_{s} \bar{U}_{t} \subset \bar{U}_{s+t}$, it follows that the geometry is strong if and only if $\bar{U}_{s} \bar{U}_{t}$ is closed. If the semigroup $S$ is archimedean in the sense that for every $u, v \in S$ there exists a natural number $n$ such that $n u>v$, and $G$ is a locally compact, then every element of a geometry over $S$ must have compact closure. It follows that any geometry over an archimedian semigroup on a locally compact group is a strong geometry.

Example 1.1. Every (connected) Lie group with invariant Riemannian or Finsler metric is a geometric group parameterized over $\mathrm{R}^{+}$, where the geometry is the collection of metric balls centered at $e$. We will show (Proposition 3.1) that any geometry over $\mathrm{R}^{+}$on a topological group corresponds to an invariant inner metric. It then follows from ([3]) that, conversely, every geometry over $\mathrm{R}^{+}$on a Lie group must come from a (possibly non-holonomic) invariant Finsler metric.

Example 1.2. Every Banach space is an example of a strong geometry over $\mathrm{R}^{+}$ on a topological vector space.

Example 1.3. In geometric group theory, the Cayley graph of a group $G$ with generating set $F$ is provided with an inner metric, where each edge is given length 1. Let $U_{a}=\{g \in G: d(e, g) \leq a\}$, where $d(e, g)$ denotes the length of the shortest edge-path joining the vertices corresponding to $e$ and $g$. Then $\left\{U_{a}\right\}$ is a geometry parameterized over the non-negative integers (where $G$ has the discrete topology). Normally, this geometry is assumed to have curvature bounded above in some sense (which we will not need, and therefore will not define in this paper).

Example 1.4. The first author proved ([5]) that any group $G$ can be realized as the fundamental group of a 2-dimensional topological space $X$ having a complete metric $d$ of curvature (in the sense of Alexandrov) bounded above by 1, with the additional property that every pair of points is joined by a minimal curve. The curvature bound implies that $X$ is locally contractible; thus we may pass to the universal covering $\widetilde{X}$, to which the metric $d$ can be lifted as a metric $\widetilde{d}$. Then by identifying $G$ with the images under the deck transformations (which are isometries of $\widetilde{X}$ ) of a single point $x$, we induce a natural geometry on $G$, which can also be considered to have curvature $\leq 1$ (again the topology is discrete).

Example 1.5. In [23] the second author showed that every locally compact, arcwise connected, metrizable group admits a geometry over $\mathrm{R}^{+}$having curvature bounded below in a certain sense. These geometries are true generalizations of invariant Riemannian metrics on connected Lie groups. See also Theorem 1.8.

Example 1.6. Let the positive reals have its usual order, and let $a \wedge b=\max \{a, b\}$ be the algebraic operation. Then the resulting ordered semigroup is denoted by $\mathrm{R}^{\max }$. Note that $\mathrm{R}^{\max }$ cannot be embedded in a group because the cancellation laws do not apply (e.g. $1 \wedge 3=2 \wedge 3$, but $1 \neq 2$ ). Also, $\mathrm{R}^{\max }$ is not archimedean (cf. the paragraph after Definition 1.1). Given a geometry $\left\{U_{r}\right\}$ over $\mathrm{R}^{\max }$ on a group $G$, each set $U_{r}$ consists of the metric ball centered at $e$ of radius $r$ with respect to some invariant metric $d$ (see Section 3 ). It is easy to see that this metric must be an ultrametric; i.e, for all $x, y, z \in G, d(x, y) \leq \max \{d(x, z), d(z, y)\}$. Conversely, 
every invariant ultrametric on a group gives rise to a geometry over $\mathrm{R}^{\mathrm{max}}$. It is clear that any geometry on a group $G$ parameterized over $\mathrm{R}^{\text {max }}$ must consist of open subgroups, with the result that $G$ is totally disconnected. In a later paper we will prove that every locally compact totally disconnected group admits such a geometry, and investigate these geometries more fully.

Example 1.7. Valuations on fields provide interesting examples of geometries. We give here a very brief description of valuations on fields in order to show that every valuation gives rise to a geometry over $\mathrm{R}^{+}$or $\mathrm{R}^{\max }$; references for valuations on fields are [10] and [18] (our terminology differs from that of the latter). Let $K$ be a field with additive identity 0 and multiplicative identity 1 . A valuation $|\cdot|$ on $K$ is a nonnegative real valued mapping on $K$ satisfying the following axioms:

1. $|x|=0$ if and only if $x=0$,

2. $|x y|=|x||y|$, and

3. $|x+y| \leq|x|+|y|$.

We assume that a valuation is non-trivial (i.e. there is some $x \in K$ such that $|x| \notin\{0,1\})$. In the terminology of Section 3 of this paper, a valuation on $K$ is equivalent to a family $\left\{U_{r}\right\}$ of open sets satisfying conditions M1-M5, and the sets $U_{r}:=\{x \in K:|x|<r\}$ are the metric balls centered at 0 of radius $r$ with respect to a left-invariant metric on $K$. The field $K$ with the topology induced by this metric is a topological field. Two valuations $|\cdot|_{1},|\cdot|_{2}$ on $K$ induce the same topology if and only if they are equivalent, i.e. for some number $c>0$, we have $|x|_{1}=|x|_{2}^{c}$ for all $x \in K$. Another equivalent condition is that, setting $V_{i}(r)=\left\{x:|x|_{i} \leq r\right\}$ $(i=1,2), V_{1}(1)=V_{2}(1)$.

A valuation is non-archimedean if the following condition holds: $\left(3^{\prime}\right)|x+y| \leq$ $\max \{|x|,|y|\}$. Condition $\left(3^{\prime}\right)$ is equivalent to the corresponding metric being an ultrametric, and so from Example 1.6, we see that $\left\{U_{r}\right\}$ must be a geometry of the (additive) group $K$ over $\mathrm{R}^{\max }$. A non-archimedean valuation on a field $F$ can be extended to a (non-archimedean) valuation on any finite dimensional extension field $E$ of $F$.

In the equivalence class of any archimedean (i.e. not non-archimedean) valuation on $K$ there is a unique valuation which defines a strong geometry over $\mathrm{R}^{+}$, on $K$; it is the geometry induced by an embedding of $K$ in the complex numbers $\mathrm{C}$ with the usual norm.

As a standard example, one can define on the rational numbers $Q$ the (nonarchimedean) $p$-adic valuation $|\cdot|_{p}$ for any prime $p$ by $\left|\frac{p^{a} u}{v}\right|_{p}=p^{-a}$, where $u, v$ are nonzero and not divisible by $p$. (We set $|0|=0$.) Every nontrivial valuation on $\mathrm{Q}$ is equivalent to $|\cdot|_{p}$ for some $p$ or the usual absolute value $|\cdot|_{\infty}$.

Every "valuated" field $K$ has a completion $\bar{K}$ : a valuated field containing $K$ whose metric is the completion of the metric of $K$. For example, the completion of $\mathrm{Q}$ with $|\cdot|_{\infty}$ is $\mathrm{R}$ with $|\cdot|_{\infty}$; the completion of $\mathrm{Q}$ with $|\cdot|_{p}$ is the field $\mathrm{Q}_{p}$ of $p$-adic numbers. The closure $\mathrm{Z}_{p}$ of $\mathrm{Z}$ in $\mathrm{Q}_{p}$ is the ring of $p$-adic integers. The extension of a complete valuation on $F$ to a finite dimensional extension $E$ of $F$ is uniquely determined.

Every local (i.e. nondiscrete locally compact) topological field is complete relative to any compatible valuation. As an example of such a field we can take the field $F_{q}((t))$ of formal power series over the finite field $F_{q}$ with $q$ elements, where $q=p^{s}, p$ a prime. Here each element $x$ of $F_{q}((t))$ and the valuation $|\cdot|_{c}$ have the 
following form: $x=0$ or $x=\sum_{n=I}^{\infty} \alpha_{n} t^{n}, I \in \mathrm{Z}, \alpha_{n} \in F_{q}, \alpha_{I} \neq 0 ;|x|_{c}=c^{I}, 0<c<1$, and $|0|=0$. Every local field admits at most one (up to equivalence) compatible valuation $|\cdot|$. Up to isomorphism there are only three possible cases, each of which corresponds to a geometry:

(a) If $|\cdot|$ is archimedean, then $(K,|\cdot|)=\left(\mathrm{R},|\cdot|_{\infty}\right)$ or $\left(\mathrm{C},|\cdot|_{\infty}\right)$.

(b) If $|\cdot|$ is non-archimedean and the characteristic of $K$ is 0 , then $K$ is a finite dimensional extension of $\mathrm{Q}_{p}$, for some prime $p$, and $|\cdot|$ is the unique extension of $|\cdot|_{p}$.

(c) If $|\cdot|$ is non-archimedean and the characteristic of $K$ is $p \neq 0$, then $(K,|\cdot|)=$ $\left(F_{q}((t)),|\cdot|_{c}\right)$ for some $q=p^{s}$ and $0<c<1$.

If $K$ is a valuated field, we let $V_{r}=\{x:|x| \leq r\}$ and note that $V:=V_{1}$ is a multiplicative semigroup of $K$. The set $U=V-U_{1}$ is the group of units, and contains 1 . If the valuation is non-archimedean, then $V$ is a ring, the so-called valuation ring.

More generally, one can define a valuation on a field $K$ with values in a linearly ordered multiplicative group $(S, \cdot, \leq)$ with unit $1,(S$ is the value group $)$ using the axioms (1), $(2),\left(3^{\prime}\right)$. It is interesting to note that every such group $S$ can be realized as the value group for an appropriate valuated field. For such a valuation, the valuation $\operatorname{ring} V$ has the property that for any $x \in K \backslash\{0\}, x$ or $x^{-1}$ is in $V$. Conversely, every such $\operatorname{ring} V$ in a field $K$ is a valuation ring for some valuation $|\cdot|$ with value group $S=K^{*} / U$. Here $K^{*}=(K \backslash\{0\}, \cdot)$ is the (abelian) multiplicative group of the field $K$ and $U$ is the group of units of the ring $V$, which consists of all $x \in K$ such that $x$ and $x^{-1}$ are both in $V$. Let $f: K^{*} \rightarrow K^{*} / U$ be the canonical automorphism. One defines $f(x) \leq f(y)$ if and only if $x y^{-1} \in V, 0 \leq x$; and $|x|=f(x), x \in K^{*},|0|=0$. Evidently one obtains in this way a geometry on the additive group $K$.

Example 1.8. Let $Z(K)$ be the integer lattice in the algebra $K$ of the real, complex, or quaternion numbers, given a Euclidean norm $\|\cdot\|$ such that $\|a b\|=\|a\|\|b\|$ and $\|1\|=1$. For example, $Z(\mathrm{R})$ is the integers; $Z(\mathrm{C})$ is the "Gaussian integers." We let $l$ be the real dimension of the vector space $K$. Then any ideal $J$ in $Z(K)$ is principal: $J=(q)$, where $q$ is an element in $J$ of least norm. Since $J$ is generated from the cyclic subgroup generated by $q$ and rotations of angle $\frac{\pi}{2}$, it is easy to see geometrically that the "fundamental domain" for such an ideal is an $l$-dimensional cube of side length $\|q\|$. It follows that the index $I(J)=[Z(K): J]=\|q\|^{l}$. For $\|q\|>1$, we can consider the ideals $J(n)=\left(q^{n}\right), n=0,1,2, \ldots$, where $\left(q^{0}\right)=Z(K)$. The natural inverse limit $G$ of the finite abelian groups $Z(K) / J(n)$ is a compact totally disconnected group, in fact a generalization of the $p$-adic integers. (Here the group operation is that induced by the sum, not the product, in the algebra $K$, so is abelian even for the quaternions.) For any $z \neq 0$ in $Z(K)$, we let $\operatorname{deg}(z)=\max \{n: z \in J(n)\}$. Then $\operatorname{deg}(z)<\infty$ since $\bigcap J(n)=\{0\}$. We define $\operatorname{deg}(0)=+\infty$. If we let $U_{r}=\{z: f(z)<r\}$, where $f(z)=\|q\|^{-\operatorname{deg}(z)}$, we obtain a geometry on $Z(K)$. Alternatively, we can take a power of $f(z)$ in the definition. If we take the completion of the metric corresponding to this geometry, we obtain a geometry on $G$ of the type described in Example 1.6.

It is interesting to observe the contrast between geometries over $\mathrm{R}^{+}$and $\mathrm{R}^{\max }$. The first requires a very connected (i.e. arcwise and locally arcwise connected) topology, while the second requires a totally disconnected topology. A geometry 
over $\mathrm{R}^{+}$corresponds to an inner metric, which for a locally compact group has the property that for every distinct $x, y$, there is a distinct point $z$ such that $d(x, y)=$ $d(x, z)+d(z, y)$. A geometry over $\mathrm{R}^{\max }$ corresponds to an ultrametric, which has the property that for distinct points $x, y, z$, the triangle inequality is never an equality.

Note that, in the above examples, if $G$ has finite diameter, then $U_{a}=G$ for sufficiently large $a$; in general it need not be true that if $r$ is strictly less than $s$, then $U_{r}$ is a proper subset of $U_{s}$. There are other types of semigroups of open sets on groups (and other algebraic objects) in which some of the conditions of Definition 1.1 have been changed or omitted, but which should also be considered as geometries or pseudogeometries of some sort. We will consider these in a later paper.

A collection of not necessarily open sets $\left\{U_{s}\right\}$ satisfying the conditions of Definition 1.1, except G3, for $S=\mathrm{R}^{+}$, has been called a Gleason semigroup in [14] . The existence of non-trivial Gleason semigroups for metrizable locally compact groups can be used to show the existence of non-trivial one-parameter subgroups as part of a solution to Hilbert's Fifth Problem (cf. [14]). In general the sets in a Gleason semigroup are not open (and in fact, by Proposition 3.1, for locally compact, connected groups, they can be open only if the underlying group is arcwise connected). We do not know of a direct argument (and such would be interesting) that, for an arcwise connected locally compact, metrizable group, the simple construction of Gleason semigroups shown in [14] can be carried out in such a way that one obtains a geometry over $\mathrm{R}^{+}$(and hence an inner metric).

In this paper the word "group" will mean a Hausdorff topological group, and $e$ always denotes the identity. A subgroup of a group is assumed to have the subspace topology. By "topological isomorphism" we mean a group isomorphism that is also a homeomorphism. At present we are concerned mainly (not exclusively) with arcwise connected groups, and the relation between topological properties and geometric ones. Since our spaces are arcwise connected, we are interested in geometries over $\mathrm{R}^{+}$. While a number of our present results concern locally compact groups, one of our goals is to remove, to whatever extent possible, the assumption of local compactness. This effort is necessary even for a proper study of locally compact groups, since the universal covering group (see Theorem 1.5) of a locally compact group may not be locally compact. We are further motivated by the fact that infinite dimensional groups (particularly transformation groups) are often not locally compact. Local compactness is a convenient assumption because it provides an invariant measure, and therefore a way to do analysis. Our goal is to use direct geometric methods in more general situations.

We begin Section 2 by introducing notions of "semidiscrete" and "hemidiscrete" groups (Definition 2.1), and define an arcwise connected group $\widetilde{G}$ to be a cover of an arcwise connected group $G$ if $G=\widetilde{G} / H$, for some closed, normal, semidiscrete subgroup $H$. Note that the quotient map $\pi: \widetilde{G} \rightarrow G$ may not be locally injective (and so $\pi$ is not a covering map in the usual sense). We prove:

Theorem 1.1. Let $G$ be a complete arcwise connected topological group and $H$ a closed, normal semidiscrete subgroup of $G$. Suppose $X$ is a simply connected, arcwise connected and locally arcwise connected space. If $f: X \rightarrow G / H$ is a (continuous) map such that $f\left(x_{0}\right)=e$, then there is a unique (continuous) lift $\tilde{f}: X \rightarrow G$ such that $\tilde{f}\left(x_{0}\right)=e$ and $\pi \circ \tilde{f}=f$, where $\pi: G \rightarrow G / H$ is the natural projection. 
Corollary 1.2. If $\widetilde{G}$ is an arcwise connected, simply connected complete cover of a complete group $G$ and $\pi: \widetilde{G} \rightarrow G$ is the quotient mapping, then $\operatorname{ker}(\pi)$ is naturally isomorphic to $\pi_{1}(G)$.

By $\pi_{1}(G)$ we mean, as usual, homotopy equivalence classes of loops based at $e$ with the usual multiplication. Since left translation is a homeomorphism, the fundamental group is independent (up to isomorphism) of base point even if the group is not arcwise connected.

Corollary 1.3. If $G$ is a complete group with complete arcwise connected, locally arcwise connected, simply connected covers $\widetilde{G}_{1}$ and $\widetilde{G}_{2}$, then $\widetilde{G}_{1}$ is topologically isomorphic to $\widetilde{G}_{2}$.

Corollary 1.4. Let $G$ be a complete group with arcwise connected, locally arcwise connected, complete covers $\widetilde{G}_{1}$ and $\widetilde{G}_{2}$. If $\widetilde{G}_{1}$ is simply connected, then there exists a cover $\pi: \widetilde{G}_{1} \rightarrow \widetilde{G}_{2}$.

Corollaries 1.3 and 1.4 justify referring to a simply connected arcwise and locally arcwise connected group $\widetilde{G}$ that covers a group $G$ as the universal cover of $G$. We don't know to what extent local arcwise connectedness is needed in the above corollaries. However, it should be pointed out that $S^{1}$ is covered both by $\mathrm{R}$ (in the usual way) and by the solenoid $\Sigma$. Recall that $\Sigma$, which is connected but not arcwise connected, is the inverse limit of circles, where the connecting maps are multiplication by 2 ; the projection of $\Sigma$ onto any of the circles can easily be shown to have hemidiscrete kernel. This example also demonstrates one difficulty in showing, in general, the existence of a universal covering group for a given arcwise connected group: an inverse limit of groups in this category, even with discrete kernels of the connecting maps, need not be arcwise connected.

In [13], Glushkov gives a construction of the universal covering of a connected, locally connected, locally compact group (this paper was apparently never translated into English). Later, Lashof also gave a different construction of the universal covering group of a connected, locally compact (or more generally $L P$-group) in [20]. Glushkov constructed the Lie algebra for a locally compact group in [12] and Lashof used a different construction of a the same object in [20]. Glushkov proved that the universal cover is uniquely determined by the Lie algebra, and Lashof proved the universal cover is independent of the particular inverse limit used in his construction. We give a shorter, alternative to construction of the GlushkovLashof cover for arcwise connected, metrizable locally compact groups, obtaining the following theorem, which adds to the Glushkov-Lashof result that the kernel of the cover is hemidiscrete (rather than just totally disconnected) and also part (5) (which we will need later). See also Definition 2.1.

Theorem 1.5. Let $G$ be a locally compact, arcwise connected, metrizable group. Then there exist

1. a simply connected Lie group $L$ ( $L$ is either trivial or non-compact),

2. at most countably many simply connected, compact, simple Lie groups $G_{i}$,

3. an at most countable product $\mathrm{R}^{\omega}$ of the reals,

4. an epimorphism $\pi: \widetilde{G}:=L \times G_{1} \times \cdots \times \mathrm{R}^{\omega} \rightarrow G$ such that $\operatorname{ker} \pi$ is hemidiscrete, and

5. the projection of $\operatorname{ker} \pi$ onto the factor $\mathrm{R}^{\omega}$ is the lattice $\mathrm{Z} \times \frac{1}{2} \mathrm{Z} \times \frac{1}{4} \mathrm{Z} \times \cdots$. 
Note that Corollary 1.3 gives a different, and in some ways more satisfactory, uniqueness result than those of Glushkov and Lashof.

Rickert ([25]) stated a path lifting lemma for locally compact groups, but his sketchy proof is partly incorrect. Rickert's result also cannot be applied to the simply connected cover of a locally compact group because (as is evident from Theorem 1.5) it may not be locally compact. It also turns out that [13] contains many of Rickert's (later) results, including the equivalence of arcwise connectedness and local connectedness for locally compact, connected groups.

In Section 3 we give a purely algebraic description of the notion of invariant metric and invariant inner metric on a group, which leads naturally to the above definition of geometric group. As we have mentioned, an invariant inner metric is essentially a geometry over $\mathrm{R}^{+}$. An invariant metric is essentially the same as a geometry over $\mathrm{R}^{+}$, except that the semigroup condition (G5) is weakened to $U_{s} \cdot U_{t} \subset U_{s+t}$ (which corresponds to the triangle inequality). To demonstrate the usefulness of this perspective we give a very simple (compared to the elsewhere repeated argument found in [21]) proof that a first countable group is metrizable. Our algebraic description also allows for the easy introduction of a quotient metric (or inner metric), which has the following useful property:

Proposition 1.6. Let $G$ be a group with invariant metric (or inner metric), and $H$ be a closed normal subgroup. If $\pi: G \rightarrow G / H$ is the quotient map, then $\pi$ is a weak submetry with respect to the quotient metric (or inner metric) on $G / H$.

The notions of weak submetry (resp. submetry) were introduced in [3]; they simply mean that any open (resp. closed) metric ball $B(p, r)$ (resp. $C(p, r)$ ) is mapped precisely to $B(\pi(p), r)$ (resp. $C(\pi(p), r)$ ). For example, a Riemannian submersion is a weak submetry, and it is possible to prove that, conversely, every weak submetry between Riemannian manifolds is a Riemannian submersion of class $C^{1}$ (these are unpublished results of the first author). We show in the present paper (Proposition 3.4) that a weak submetry preserves a lower curvature bound. We immediately have:

Corollary 1.7. If $G$ is a group with invariant inner metric of curvature $\geq k$ and $H$ is a closed normal subgroup of $G$, then the quotient metric on $G / H$ is an invariant inner metric of curvature $\geq k$.

We will not specifically use the property of curvature $\geq k$ in this paper, except in the proof of Proposition 3.4; for further information and definitions we refer to [23]. We point out here that if $H$ is compact in Proposition 1.6, then $\pi$ is in fact a submetry. Also, we can use essentially the same definition to define a more general quotient geometry, but we won't use this idea in the present paper.

We give an application of Corollary 1.7 in Section 3: First we show that for a locally compact, arcwise connected, metrizable group $G$, there is a surjective homomorphism $\pi^{\prime}: L \times G_{1} \times \cdots \times E \rightarrow G$, where $E=\mathrm{R}^{\omega}$ when $\omega$ is finite, $E=l^{2}$ is (the natural embedding of) separable Hilbert space in $R^{\omega}$ when $\omega$ is infinite, and $\pi^{\prime}$ is the restriction of the map $\pi$ from Theorem 1.5. We call $\pi^{\prime}$ the metric restriction of $\pi$. We prove the following theorem, which strengthens, and simplifies the proof of, the main existence theorem of [23]:

Theorem 1.8. Let $G$ be an arcwise connected, first countable, locally compact group, and consider the metric restriction $\pi^{\prime}: \widetilde{G}_{M}=L \times G_{1} \times \cdots \times E \rightarrow G$ 
of the universal cover $\pi: \widetilde{G} . \rightarrow G$. If the factors of $\widetilde{G}_{M}$ are given appropriately selected Riemannian metrics and $\widetilde{G}_{M}$ is given the product metric, the quotient metric on $G=\widetilde{G}_{M} / \operatorname{ker} \pi^{\prime}$ is compatible with the topology of $G$, and is an invariant inner metric of curvature $\geq k$.

The relevant definitions may be found in Section 3. Note that the topology on $\widetilde{G}_{M}$ is finer than the subspace topology induced by $\widetilde{G}$ because the inclusion $E \hookrightarrow R^{\omega}$ is continuous, but if $E$ is infinite dimensional, the inclusion is not a homeomorphism onto its image. Nonetheless the quotient topology on $\widetilde{G}_{M} / \operatorname{ker} \pi^{\prime}$ is the same as that of $G$. This is possible because the "fundamental domain" of the mapping $\pi^{\prime}$ may have no interior. For example, in the special case of $G=T^{\infty}=S^{1} \times S^{1} \times \cdots$, the fundamental domain is the Hilbert cube in $l^{2}$, and the mapping $\pi^{\prime}: l^{2} \rightarrow T^{\infty}$ can be considered as identifying "opposite sides" of this (compact) domain. Note also that since the topology on $\widetilde{G}_{M}$ is finer than the induced topology, $\operatorname{ker} \pi^{\prime}=\operatorname{ker} \pi \cap \widetilde{G}_{M}$ is totally disconnected. On the other hand, $l^{2}$ is not topologically isomorphic to $\mathrm{R}^{\infty}$ (for example, no finite metric ball in $l^{2}$ contains a 1-parameter subgroup, whereas every basis element of $\mathrm{R}^{\infty}$ does). (Recall that Anderson and Bing proved that $l^{2}$ and $\mathrm{R}^{\infty}$ are homeomorphic in [1].) Therefore, from Corollary 1.3 and the fact that a closed subgroup of a complete group is complete, we obtain the following:

Proposition 1.9. The kernel of the natural mapping $\pi^{\prime}: l^{2} \rightarrow T^{\infty}$ is complete and totally disconnected but not semidiscrete.

Thus we see that merely having a totally disconnected kernel is not sufficient for a continuous homomorphism to have adequate covering properties. In the next paragraph we will see explicitly which curves cannot be lifted in $\pi^{\prime} ; \operatorname{ker} \pi^{\prime}$ is only a special subgroup of $\pi_{1}\left(T^{\infty}\right)$ explicitly described by the metric we have constructed.

In a compact Riemannian manifold every free homotopy class of closed curves contains a shortest representative, and that representative is a closed geodesic (if we fix a base point, the same result holds, but the shortest element is only a geodesic loop, with a possible break at the base point). The proof is roughly this: Due to the local convexity of the space, one can always find a piecewise geodesic in every path homotopy class. In particular, we can find a rectifiable curve in the class, and so by using Ascoli's theorem (and the local convexity) we can find a shortest curve in the class. If we suppose this shortest curve is not a closed geodesic, we can use local convexity a third time to shorten it, and obtain a contradiction. A similar claim was made in [15] for compact inner metric spaces, but the statement given there is not true. In Section 4 we give a simple counterexample contained in the plane, in which a path homotopy class has a shortest element but does not contain a geodesic. More importantly, we show that similar behavior occurs in the infinite torus $T^{\infty}=S^{1} \times S^{1} \times \cdots$ with a product metric, and moreover there are path homotopy classes containing no rectifiable curves at all. We can now see explicitly why $\pi^{\prime}$ does not have good covering properties: with the metric induced by $\pi^{\prime}$ (via Theorem 1.8), it is not possible to lift non-rectifiable curves to $\widetilde{G}_{M}$, so path homotopy classes without rectifiable curves are not represented in ker $\pi^{\prime}$. We note here that the third author has shown ([27]) using Theorem 1.8 that given any locally compact, metrizable, arcwise connected group $G$ and 1-parameter subgroup $\gamma$, one can construct an invariant inner metric of curvature bounded below such that $\gamma$ is rectifiable.

Our last result in this paper is the following: 
Theorem 1.10. Let $G$ be an arcwise connected, locally compact, metrizable group equipped with a metric of the form given by Theorem 1.8. Then every path homotopy class based at e contains a curve of the form $c(t)=\gamma(t) \omega(t)=\omega(t) \gamma(t)$, where $\gamma(t)$ is a geodesic and $\omega(t)$ is a 1-parameter subgroup $(\gamma(t)$ or $\omega(t)$ may be trivial).

\section{Simply CONNECTED AND UNIVERSAL COVERING GROUPS}

A net in a topological space is a subset $\left\{x_{\alpha}\right\}_{\alpha \in A}$ indexed on some directed set $A$ (i.e., a net is a kind of generalized sequence for spaces which do not have a countable basis). Convergence has essentially the same meaning for nets as it does for sequences. A net $\left\{x_{\alpha}\right\}_{\alpha \in A}$ in a topological group is Cauchy if for every neighborhood $U$ of $e$ there exists an $\alpha$ such that for all $\beta \geq \alpha$ in $A, x_{\beta}^{-1} x_{\alpha} \in U$. A topological group is complete if every Cauchy net is convergent. Locally compact groups and groups admitting a metrically complete invariant metric are complete; every invariant metric on a complete group must be (metrically) complete. Not every (non-abelian) topological group can be completed as a topological group (see [7] for more details). If $G$ is a topological group and $A$ is a directed indexing set, an ordered family $\left\{K_{\alpha}\right\}_{\alpha \in A}$ of (closed) subgroups of $G$ is a set of closed subgroups such that if $\alpha<\beta$, then $K_{\beta} \subset K_{\alpha}$. Associated with an ordered family $\left\{K_{\alpha}\right\}_{\alpha \in A}$ is an inverse system of maps $G / K_{\alpha} \stackrel{\pi_{\alpha \beta}}{\leftarrow} G / K_{\beta}$. Recall that the inverse limit $\lim _{\longleftarrow} G / K_{\alpha}$ is the subset of the product $\prod_{\alpha \in A} G / K_{\alpha}$ consisting of those elements $\left(g_{\alpha} \overleftarrow{\left.K_{\alpha}\right)} \text { }\right)_{\alpha \in A}$ such that $g_{\alpha} K_{\alpha}=\pi_{\alpha \beta}\left(g_{\beta} K_{\beta}\right)$; i.e., $g_{\alpha} g_{\beta}^{-1} \in K_{\alpha}$. If each $K_{\alpha}$ is normal, then $\lim _{\longleftarrow} G / K_{\alpha}$ has the structure of a group. There is a natural mapping $g \mapsto\left(g K_{\alpha}\right)$ of $G$ into $\underset{\lim }{\longleftarrow} / K_{\alpha}$, which is a homomorphism if each $K_{\alpha}$ is normal. The natural mapping is injective if and only if $\bigcap_{\alpha \in A} K_{\alpha}=\{e\}$. For example, if $G$ has an ordered family $\left\{K_{\alpha}\right\}$ such that $G / K_{\alpha}$ is discrete and $\bigcap_{\alpha \in A} K_{\alpha}=\{e\}$, then $G$ embeds in the totally disconnected product $\prod_{\alpha \in A} G / K_{\alpha}$, and so is itself totally disconnected. The following lemma is proved in [7] for normal subgroups; the proof of the statement below can be found in [27].

Lemma 2.1. Let $G$ be complete and $\left\{K_{\alpha}\right\}_{\alpha \in A}$ be an ordered family of subgroups of $G$ such that for every open $U \ni$ e there exists an $\alpha$ such that $K_{\alpha} \subset U$. Then the natural mapping $i: G \longrightarrow \lim G / K_{\alpha}$ is 1-1 and onto. Thus if each $K_{\alpha}$ is normal, $i$ is a topological isomorphism.

Definition 2.1. A complete topological group $H$ is called semidiscrete (resp. hemidiscrete) if every neighborhood of $e$ in $H$ contains an open (resp. open normal) subgroup.

Clearly any discrete group is hemidiscrete, and the notions of hemidiscrete and semidiscrete are equivalent for abelian groups. Recall that an open subgroup of a topological group is automatically closed (cf. [24], p. 102). If $K$ is an open (and hence closed) subgroup of a topological group $H$, then $H / K$ is discrete. By the above comments we now immediately have:

Lemma 2.2. A group $G$ is semidiscrete (resp. hemidiscrete) if and only if it is the inverse limit of discrete $G$-spaces (resp. groups).

Recall that a space $X$ is a $G$-space if $G$ acts transitively on $X$. If $H$ is semidiscrete, then by Lemma $2.2, H$ can be written as an inverse limit of discrete spaces 
and is therefore totally disconnected. Proposition 1.9 shows that the converse statement is false. On the other hand, it follows from results in [24], p. 131 that a locally compact totally disconnected group must be semidiscrete. The proof of the next lemma is easy.

Lemma 2.3. Let $G$ be a group.

1. If $G$ is semidiscrete (resp. hemidiscrete), then any closed subgroup of $G$ is semidiscrete (resp. hemidiscrete).

2. If $G$ is semidiscrete (resp. hemidiscrete) and $H$ is a closed normal subgroup of $G$, then $G / H$ is semidiscrete (resp. hemidiscrete).

3. If $G$ is the direct product (possibly infinite) or inverse limit of semidiscrete (resp. hemidiscrete) groups, then $G$ is semidiscrete (resp. hemidiscrete).

Proposition 2.4. Suppose $G$ is a complete group, $H$ is a closed, central subgroup of $G$, and both $H$ and $G / H$ are hemidiscrete. Then $G$ is semidiscrete.

Proof. Let $U$ be an open neighborhood of $e$ in $G$. Since $H$ is hemidiscrete, there is an open set $V$ contained in $U$ such that $V \cap H$ is an open normal subgroup $N$ of $H$. Since $H$ is central, $N$ is also normal in $G$. By the Third Isomorphism Theorem, the kernel of the natural map $\pi: G / N \rightarrow G / H$ is isomorphic to $H / N$, which is a discrete group. Thus $\pi$ is a local isomorphism. Since $G / N$ is locally isomorphic to the hemidiscrete group $G / H, G / N$ is semidiscrete. The proof is now complete by Lemmas 2.1 and 2.3 .

Example 2.1. Let $H=\mathrm{Z} /(2) \times \mathrm{Z} /(2) \times \cdots$ and $G$ be the semidirect product of $H$ by $\mathrm{Z}$, with multiplication given by

$$
\left(m,\left(a_{i}\right)\right)\left(n,\left(b_{i}\right)\right)=\left(m+n,\left(a_{i+n}+b_{i}\right)\right) .
$$

That is, $\mathrm{Z}$ acts on $H$ by translating the coordinates of each element. Then it is not hard to show that if $h:=\left(n,\left(b_{i}\right)\right) \in G$ is non-trivial such that $b_{k}=0$ for some $k$, then there exists a $g \in G$ such that $\left(s,\left(c_{i}\right)\right):=g^{-1} h g$ satisfies $c_{k} \neq 0$. It follows that any nontrivial subgroup of $H$ that is normal in $G$ must equal $H$. So even though $H$ and $G / K \equiv \mathrm{Z}$ are hemidiscrete, $G$ is not.

Lemma 2.5. If $H$ is a totally disconnected normal subgroup of an arcwise connected group $G$, then $H$ is central.

Proof. Let $h \in H$ and let $g \in G$. We need to show that $g^{-1} h g=h$. Since $G$ is arcwise connected there is an arc $\gamma$ from $e$ to $g$. Let $\alpha$ be the arc given by $\alpha(t)=(\gamma(t))^{-1} h \gamma(t)$. Then $\alpha$ is an arc from $e$ to $g^{-1} h g$ that stays in $H$. But $H$ being totally disconnected can contain no nontrivial arcs. Hence $\alpha$ must be the arc that is constantly $h$ and we obtain that $g^{-1} h g=h$.

Corollary 2.6. Any semidiscrete subgroup of an arcwise connected group is central and hemidiscrete.

Proposition 2.7. Let $A, B, C$ be complete arcwise connected groups and suppose that there are covers $\pi_{1}: A \rightarrow B$ and $\pi_{2}: B \rightarrow C$. Then $\pi_{2} \circ \pi_{1}: A \rightarrow C$ is a cover.

Proof. By Corollary $2.6 \operatorname{ker} \pi_{i}$ is central and hemidiscrete for $i=1,2$. The proof is now complete by letting $G=\operatorname{ker}\left(\pi_{2} \circ \pi_{1}\right), H=\operatorname{ker} \pi_{1}$ and $K=\operatorname{ker} \pi_{2}$ in Proposition 2.4, and using Corollary 2.6. 
A closed, semidiscrete subgroup $H$ of a Lie group $G$, being totally disconnected, is in fact discrete. This means that the quotient map $G \rightarrow G / H$ is a covering map. Theorem 1.1 is a generalization of this fact.

Proof of Theorem 1.1. By Corollary 2.6, $H$ is hemidiscrete and central, and we can take open normal subgroups $\left\{K_{\alpha}\right\}$ of $H$, which constitute a basis for the topology of $H$ at $e$. By Lemma 2.1, $H=\varliminf_{H} H / K_{\alpha}$; each $H / K_{\alpha}$ is a discrete group. Since $H$ is central, each $K_{\alpha}$ is central, and hence normal in both $H$ and $G$. There is a natural topological isomorphism, $\phi_{\alpha}: G / H \rightarrow Q_{\alpha}:=\left(G / K_{\alpha}\right) /\left(H / K_{\alpha}\right)$. Now let $f_{\alpha}=\phi_{\alpha} \circ f: X \rightarrow Q_{\alpha}$. Since the natural map $q_{\alpha}: G / K_{\alpha} \rightarrow Q_{\alpha}$ has discrete kernel, it is a covering map (cf. [24], p. 360). Since $X$ is simply connected, arcwise connected and locally arcwise connected, $f_{\alpha}$ has a unique lift $\widetilde{f}_{\alpha}: X \rightarrow G / K_{\alpha}$ such that $\tilde{f}_{\alpha}\left(x_{0}\right)=K_{\alpha}$ and $q_{\alpha} \circ \tilde{f}_{\alpha}=f_{\alpha}$. (For details on lifting maps to covers, see [22], Lemma 14.2.)

Let $\tilde{f}: X \rightarrow \prod G / K_{\alpha}$ be defined by $\widetilde{f}(x)=\left(\widetilde{f}_{\alpha}(x)\right)_{\alpha \in A}$. We first claim that $\pi_{\alpha \beta} \circ \widetilde{f}_{\beta}=\widetilde{f}_{\alpha}$, where $\pi_{\alpha \beta}: G / K_{\beta} \rightarrow G / K_{\alpha}, \alpha<\beta$, is the natural map. By uniqueness we need to show that $q_{\alpha} \circ \pi_{\alpha \beta} \circ \widetilde{f}_{\beta}=f_{\alpha}$. If $f(x)=g H$, then $f_{\beta}(x)=$ $\left\{g K_{\beta}\right\}$ (equivalence class $\bmod H$ ) and $\widetilde{f}_{\beta}(x)=g_{\beta} K_{\beta}$, with $g_{\beta}^{-1} g \in H$. Now $q_{\alpha}$ 。 $\pi_{\alpha \beta} \circ \tilde{f}_{\beta}(x)=q_{\alpha}\left(g_{\beta} K_{\alpha}\right)=\left\{g_{\beta} K_{\alpha}\right\}=f_{\alpha}(x)$ (since $g_{\beta}^{-1} g \in H$ ), and the claim is proved. An immediate consequence of the claim is that $\widetilde{f}(x) \in \lim G / K_{\alpha}$; i.e., we have in fact defined a mapping $\tilde{f}: X \rightarrow \varliminf_{\lim } G / K_{\alpha}$, which is isomorphic to $G$, by Lemma 2.1.

We next claim that $\pi \circ \tilde{f}=f$. As in the previous paragraph, we have, for $x \in G, f(x)=g H$ and $\widetilde{f}(x)=\left(g_{\alpha} K_{\alpha}\right)_{\alpha \in A}$, where $g_{\alpha}^{-1} g \in H$ for all $\alpha$. Since $\widetilde{f}(x) \in G=\lim G / K_{\alpha}$, we can also write $\widetilde{f}(x)=g^{\prime}=\left(g^{\prime} K_{\alpha}\right)_{\alpha \in A}$; i.e., for all $\alpha$, $g_{\alpha}^{-1} g^{\prime} \in K_{\alpha} \subset H$; whence $g^{-1} g^{\prime} \in H$, and the claim follows.

The continuity of $\widetilde{f}$ and the fact that $\widetilde{f}\left(x_{0}\right)=e$ are immediate from the definition. Uniqueness follows from the total disconnectedness of $H$. We proceed as in [25]. If $\widetilde{g}$ is another lift, let $Z=\left\{(\widetilde{g}(x))^{-1}(\widetilde{f}(x)): x \in X\right\}$. Since $\pi(\widetilde{g}(x))=\pi(\widetilde{f}(x))$, $Z \subset H$. But $Z$ is connected, so $Z=\{e\}$.

Proof of Corollary 1.2. We define a map $\xi: \pi_{1}(G) \rightarrow \operatorname{ker} \pi$ in the usual way: $\xi([\alpha])=\widetilde{\alpha}(1)$ where $\widetilde{\alpha}$ is the lift of $\alpha:[0,1] \rightarrow G(\alpha(0)=\alpha(1)=e)$ starting at $e$. It is trivial to verify that $\xi$ is well-defined and injective. To see that $\xi$ is a homomorphism, recall that, if $*$ denotes concatenation, $[\alpha * \beta]=[\alpha \beta]$. Let $\widetilde{\alpha}, \widetilde{\beta}, \widetilde{\alpha \beta}$ be the lifts of $\alpha, \beta$, and the product $\alpha \beta$, to $\widetilde{G}$ at $e$. Then $\pi\left(\widetilde{\alpha \beta}(t)\left(\widetilde{\beta}(t)^{-1} \widetilde{\alpha}(t)^{-1}\right)\right)=$ $\alpha(t) \beta(t) \beta(t)^{-1} \alpha(t)^{-1}=e$ for all $t$. Hence the entire path $\widetilde{\alpha \beta}(t)\left(\widetilde{\beta}(t)^{-1} \widetilde{\alpha}(t)^{-1}\right)$ lies in $\operatorname{ker} \pi$. Since $\operatorname{ker} \pi$ is totally disconnected, $\widetilde{\alpha \beta}(t)\left(\widetilde{\beta}(t)^{-1} \widetilde{\alpha}(t)^{-1}\right)=e$ for all $t$. Finally, it is immediate from the fact that $\widetilde{G}$ is arcwise connected that $\xi$ is surjective.

Proof of Corollary 1.3. By applying Theorem 1.1 to the quotients $\pi_{1}: \widetilde{G}_{1} \rightarrow G$ and $\pi_{2}: \widetilde{G}_{2} \rightarrow G$ we easily see that the lifts $p_{1}: \widetilde{G}_{1} \rightarrow \widetilde{G}_{2}$ and $p_{2}: \widetilde{G}_{2} \rightarrow \widetilde{G}_{1}$, respectively are continuous, surjective maps, and $p_{1}^{-1}=p_{2}$; i.e., $p_{1}$ is a homeomorphism. We need to see that $p_{1}$ is a homomorphism. Let $g, h \in \widetilde{G}_{1}$ and consider the product $x=p_{1}(g h) p_{1}(h)^{-1} p_{1}(g)^{-1} \in \widetilde{G}_{2}$. Then $\pi_{2}(x)=e$, so $x \in \operatorname{ker} \pi_{2}$. The 
map $\eta: \widetilde{G}_{1} \times \widetilde{G}_{1} \rightarrow \widetilde{G}_{2}$ defined by $\eta\left((g, h)=p_{1}(g h) p_{1}(h)^{-1} p_{1}(g)^{-1}\right.$ is a continuous map from the connected space $\widetilde{G}_{1} \times \widetilde{G}_{1}$ into a connected subset of the totally disconnected subgroup ker $\pi_{2}$. Since $\eta((e, e))=e, p_{1}$ must be a homomorphism.

Proof of Corollary 1.4. Let $\pi_{i}: \widetilde{G}_{i} \rightarrow G$ be the given covers for $i=1,2$. Again we apply Theorem 1.1 and lift $\pi_{1}$ to a map $\pi: \widetilde{G}_{1} \rightarrow \widetilde{G}_{2}$, which as in the above proof is a continuous surjective homomorphism. Since $\pi_{1}=\pi_{2} \circ \pi$, ker $\pi$ is a closed subgroup of $\operatorname{ker} \pi_{1}$ and so is semidiscrete.

We now begin the construction of the simply connected cover of a locally compact, arcwise connected, metric group.

Lemma 2.8. Let $G_{1}$ and $G_{2}$ be compact connected Lie groups and $p: G_{2} \rightarrow G_{1}$ be an epimorphism. Let $\widetilde{G}_{1}$ and $\widetilde{G}_{2}$ be the universal covering groups of $G_{1}, G_{2}$, respectively. Then $\widetilde{G}_{1}=S_{1} \times \cdots \times S_{m} \times \mathrm{R}^{k}$ and $\widetilde{G}_{2}=S_{1} \times \cdots \times S_{n} \times \mathrm{R}^{l}$, where each $S_{i}$ is a (compact) simple, simply connected Lie group, $m \leq n$ and $k \leq l$. There is a commutative diagram of epimorphisms

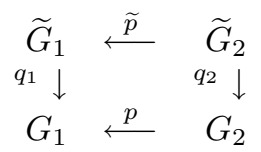

where $q_{i}$ is the covering map, and $\widetilde{p}$ is the natural projection of the product.

Proof. Let $\widetilde{p}$ denote the unique lift to $\widetilde{G}_{1}$ of the map $p \circ q_{2}$ such that $\widetilde{p}(e)=e$ and the diagram commutes. Then $\widetilde{p}$ is an epimorphism. In fact, for any $a, b \in \widetilde{G}_{2}$, $g=\widetilde{p}(a b) \widetilde{p}(b)^{-1} \widetilde{p}(a)^{-1}$ lies in ker $q_{1}$, which is discrete. For any curves $a(t), b(t)$ joining $e$ to $a$ and $b$, the curve $g(t)=\widetilde{p}(a(t) b(t)) \widetilde{p}(b(t))^{-1} \widetilde{p}(a(t))^{-1}$ joins $e$ to $g(1)$ in ker $q_{1}$, hence $g=g(1)=e$, and it follows that $\widetilde{p}$ is a homomorphism. For any $x \in \widetilde{G}_{1}$, we can connect $x$ to $e$ by a curve, project the curve to a curve $\gamma$ in $G_{1}$. Since $p$ is a fibration ([26]), we can lift $\gamma$ (perhaps not uniquely!) to $G_{2}$, then lift it again to a curve $\alpha$ connecting $e$ to some point $y \in \widetilde{G}_{2}$. Since $\widetilde{p} \circ \alpha$ must also be a lift of $q_{1} \circ \gamma$, it follows from uniqueness that $\widetilde{p}(y)=x$.

It is well-known that $\widetilde{G}_{1}$ and $\widetilde{G}_{2}$ are of the form $\widetilde{G}_{1}=S_{1} \times \cdots \times S_{m} \times \mathrm{R}^{k}$ and $\widetilde{G}_{2}=S_{1}^{\prime} \times \cdots \times S_{n}^{\prime} \times \mathrm{R}^{l}$, where each $S_{i}, S_{i}^{\prime}$ is a (compact) simple, simply connected Lie group, and this decomposition is unique. (See, for example, [16], section II.6, and use the fact that a Lie algebra determines a unique simply connected (connected) Lie group.) Since the Euclidean factor $\mathrm{R}^{j}$ in each case is the identity component of the center, it follows that $\tilde{p}\left(\mathrm{R}^{l}\right) \subset \mathrm{R}^{k}$. Likewise, if $L_{1}:=S_{1} \times \cdots \times S_{m}, L_{2}=S_{1}^{\prime} \times \cdots \times S_{n}^{\prime}$, the compactness of $L_{2}$ implies that the projection of $\widetilde{p}\left(L_{2}\right)$ onto the $\mathrm{R}^{k}$ factor must be trivial; i.e. $\widetilde{p}\left(L_{2}\right) \subset L_{1}$. It follows that if $p_{R}: \mathrm{R}^{l} \rightarrow \mathrm{R}^{k}$ and $p_{L}: L_{2} \rightarrow L_{1}$ are the restrictions of $\widetilde{p}$ to the two factors, then $\widetilde{p}=p_{R} \times p_{L}$. By rewriting the product, if necessary, we can take $p_{R}$ to be the natural projection. (In fact, $\operatorname{ker} p_{R}$ is connected by the homotopy sequence for the fibration (cf. [26]), and closed, so must be a Euclidean subgroup.) Now let $l_{2}$ be the Lie algebra of $L_{2}$. First note that we may write $l_{2}=\operatorname{ker} d p_{L} \oplus P$ where $P=\left(\operatorname{ker} d p_{L}\right)^{\perp}$ is the ideal of elements orthogonal to ker $d p_{L}$ with respect to the Killing form (see [16], Proposition 6.1). But $l_{2}$ is uniquely represented as $s_{1} \oplus \cdots \oplus s_{n}$ where $s_{i}$ is the Lie algebra of $S_{i}^{\prime}$ and Corollary 6.3 of [16] implies that $P=s_{\sigma(1)} \oplus \cdots \oplus s_{\sigma(k)}$ and $\operatorname{ker} d p_{L}=s_{\sigma(k+1)} \oplus \cdots \oplus s_{\sigma(n)}$ where $\sigma$ is some permutation of the numbers $1, \ldots, n$. Let $l_{1}$ be the Lie algebra of $L_{1}$. Then $l_{1}=d p_{L}\left(l_{2}\right)=l_{2} / \operatorname{ker} d p_{L}=P=s_{\sigma(1)} \oplus \cdots \oplus s_{\sigma(k)}$ and $d p_{L}$ is the 
projection of the direct sum $s_{1} \oplus \cdots \oplus s_{n}$ onto $s_{\sigma(1)} \oplus \cdots \oplus s_{\sigma(k)}$. It follows now from uniqueness for simply connected Lie groups that $m=k$ and that $S_{i}=S_{\tau(\sigma(i))}^{\prime}$ for $i=1, \ldots, k$ and some permutation $\tau$ of $\{\sigma(1), \ldots, \sigma(k)\}$. Uniqueness also implies that $p_{L}$ is the projection of $S_{1}^{\prime} \times \cdots \times S_{n}^{\prime}$ onto $S_{\tau(\sigma(1))}^{\prime} \times \cdots \times S_{\tau(\sigma(k))}^{\prime}$.

Theorem 2.9. Let $G$ be a compact, first countable, arcwise connected group. Then there is a commutative diagram

$$
\begin{array}{cccccccccc}
\widetilde{G}_{1} & \stackrel{\tilde{p}_{1,2}}{\longleftarrow} & \widetilde{G}_{2} & \stackrel{\tilde{p}_{2,3}}{\longleftarrow} & \ldots & \stackrel{\tilde{p}_{i-1, i}}{\longleftarrow} & \widetilde{G}_{i} & \stackrel{\tilde{p}_{i, i+1}}{\longleftarrow} & \ldots & \widetilde{G}=\lim _{q} \widetilde{G}_{i} \\
q_{1} \downarrow & & q_{2} \downarrow & & & & q_{i} \downarrow & & & \\
G_{1} & \stackrel{p_{1,2}}{\longleftarrow} & G_{2} & \stackrel{p_{2,3}}{\longleftarrow} & \ldots & \stackrel{p_{i-1, i}}{\longleftarrow} & G_{i} & \stackrel{p_{i, i+1}}{\longleftarrow} & \ldots & G=\lim G_{i}
\end{array}
$$

where each $G_{i}$ is a compact Lie group, $\widetilde{G}_{i}$ is its universal cover, $q_{i}$ is the covering map, $\operatorname{ker} p_{i-1, i}$ is connected, and

1. $\widetilde{G} \simeq S_{1} \times S_{2} \times \cdots \times \mathrm{R} \times \mathrm{R} \times \cdots$, where each $S_{i}$ is a compact, simply connected simple Lie group, $R$ is the real line, and either product, or both, may be infinite,

2. $q$ is a continuous epimorphism, and $\operatorname{ker} q=\lim \operatorname{ker} q_{i}=\lim _{\longleftarrow} \pi_{1}\left(G_{i}\right)=\pi_{1}(G)$,

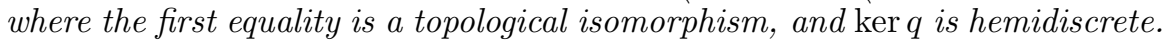

Proof. The bottom line of the diagram follows from [4], where in fact this was proved for connected, locally connected groups (which is equivalent to arcwise connectedness for locally compact groups). (Except for the connectedness of ker $p_{i-1, i}$ - which is vital for our proof - the bottom line follows from the classical theory of compact groups.) The top line and part (1) are now a direct consequence of Lemma 2.8. The mapping $q$ is defined by $q\left(\left(\widetilde{g}_{1}, \widetilde{g}_{2}, \ldots\right)\right)=\left(q_{1}\left(\widetilde{g}_{1}\right), q_{2}\left(\widetilde{g}_{2}\right) \ldots\right), \widetilde{g}_{i} \in \widetilde{G}_{i}$. Clearly $q$ is a homomorphism. To prove surjectivity, let $\left(g_{1}, \ldots\right) \in \underbrace{\lim } G_{i}=G, g_{i} \in$ $G_{i}$. Pick $\widetilde{g}_{1} \in q_{1}^{-1}\left(g_{1}\right)$. Now suppose we have chosen $\widetilde{g}_{n} \in q_{n}^{-1}\left(g_{n}\right)$ such that $\widetilde{p}_{n-1, n}\left(\widetilde{g}_{n}\right)=\widetilde{g}_{n-1}$ for all $n \leq i$. Since the fiber of $p_{i, i+1}$ is connected, it follows from the homotopy exact sequence for the fibration that the induced map $\pi_{1}\left(G_{i+1}\right) \rightarrow \pi_{1}\left(G_{i}\right)$, and hence the restriction of $\widetilde{p}_{i, i+1}$ to $\operatorname{ker} q_{i+1}=\pi_{1}\left(G_{i+1}\right)$, is surjective. Now let $\widetilde{h}_{i+1} \in q_{i+1}^{-1}\left(g_{i+1}\right)$. Then $k=\widetilde{g}_{i} \widetilde{p}_{i, i+1}\left(\widetilde{h}_{i+1}\right)^{-1} \in \operatorname{ker} q_{i}$, so there exists a $k^{\prime} \in \operatorname{ker} q_{i+1}$ such that $\widetilde{p}_{i, i+1}\left(k^{\prime}\right)=k$. Letting $\widetilde{g}_{i+1}=k^{\prime} \widetilde{h}_{i+1}$ it is easy to verify that $\widetilde{p}_{i, i+1}\left(\widetilde{g}_{i+1}\right)=\widetilde{g}_{i}$ and $q_{i+1}\left(\widetilde{g}_{i+1}\right)=g_{i+1}$. By induction, we then find $\left(\widetilde{g}_{1}, \ldots\right) \in \widetilde{G}$ such that $q\left(\left(\widetilde{g}_{1}, \ldots\right)\right)=\left(g_{1}, \ldots\right)$. It is also immediate that $\operatorname{ker} q=\lim \operatorname{ker} q_{i}=\lim _{\longleftarrow} \pi_{1}\left(G_{i}\right)$. There the first equality means that they are in fact one and the same subgroup of $\lim _{i}$; i.e. they are topologically isomorphic. The last isomorphism $\lim _{\longleftarrow} \pi_{1}\left(G_{i}\right)=\pi_{1}(G)$ follows from the homotopy exact sequence for the inverse limit. In fact, we have the short exact sequence $0 \rightarrow \lim ^{1} \pi_{2}\left(G_{i}\right) \rightarrow \pi_{1}(G) \rightarrow \lim _{\longleftarrow} \pi_{1}\left(G_{i}\right) \rightarrow 0$ (cf. [8], p. 249), and since $\pi_{2}\left(G_{i}\right)=0$ ([17], p. 93), the desired isomorphism is obtained. (We will not explain the term $\lim ^{1} \pi_{2}\left(G_{i}\right)$ here, but the unfamiliar reader should find it reasonable that it vanishes, whatever it is, if every $\pi_{2}\left(G_{i}\right)$ vanishes).

The epimorphism $q$ is continuous because of the definition of inverse limit; hence $\operatorname{ker} q$ is complete as a closed subgroup of the complete group $\widetilde{G}=\lim \widetilde{G}_{i}$. Therefore $\operatorname{ker} q=\lim \operatorname{ker} q_{i}$ is hemidiscrete.

Proof of Theorem 1.5. Suppose first that $G$ is compact. Parts (1)-(4) are immediate from Theorem 2.9. Let $Z$ be the projection of ker $q$ onto the factor $\mathrm{R}^{\omega}$. Since 
the remaining factor of this product is compact, the projection onto $\mathrm{R}^{\omega}$ is closed, so $Z$ is a closed, totally disconnected subgroup of $\mathrm{R}^{\omega}$. By [9] there exists a topological isomorphism $\phi: \mathrm{R}^{\omega} \rightarrow \mathrm{R}^{\omega}$ such that $\phi(Z)=\mathrm{Z} \times \frac{1}{2} \mathrm{Z} \times \cdots$. We extend $\phi$ to be the identity on the remaining factors of $\widetilde{G}$, and define the new mapping $\pi: \widetilde{G} \rightarrow G$ by $\pi=q \circ \phi^{-1}$. It is easy to see that $\pi$ has the desired property (5).

A simple but apparently rarely stated consequence of the Gleason-IwasawaYamabe theory of locally compact groups is that every connected locally compact group is covered (in the usual sense) by a product of a compact group and a Lie group (cf. [6], and Theorem 6, [13]). Thus the proof of the theorem is now finished by Proposition 2.7 .

\section{INVARIANT METRICS}

It is well known (cf. [21]) that a topological group $G$ admits a left-invariant metric (with the same topology) if and only if $G$ is (Hausdorff and) first countable. If $d$ is such a metric on $G$, then the family $\left\{U_{s}\right\}$ of all metric balls in $(G, d)$ of positive radius $s$ centered at $e \in G$ has the following properties for all $s, t \in \mathrm{R}$ :

M1: $U_{s}$ is an open neighborhood of $e$,

M2: $\bigcap_{s \in \mathrm{R}^{+}} U_{s}=\{e\}$, $s \in \mathrm{R}^{+}$

M3: $\bigcup_{s \in \mathrm{R}^{+}}^{s \in \mathrm{R}^{+}} U_{s}=G$,

M4: $U_{s}$ is symmetric (i.e., $U_{s}^{-1}=U_{s}$ ), and

M5: $U_{s} \cdot U_{t} \subset U_{s+t}$.

Then $d$ is determined uniquely by the formula

$$
d(g, h)=\inf \left\{s>0: h^{-1} g \in U_{s}\right\}
$$

Conversely, if any family of sets $\left\{U_{s}\right\}$ has properties M1-M5, then formula 3.1 defines an invariant metric $d$ on $G$ such that $U_{s}$ is the metric ball of radius $s$ centered at $e$. If the sets $\left\{U_{s}\right\}$ form a basis for the topology of $G$ at $e$, then the topology of $d$ agrees with the topology of $G$. Note that we allow $U_{s}$ to be defined for all positive $s$ even if the diameter of $G$ is finite.

We now argue the existence of a left invariant metric for a first countable group $G$. First, there exists a family $\left\{U_{s}\right\}$, with $s=2^{-n}(n=0,1, \ldots)$ satisfying M1-M4 having the property that if $s_{1}+\cdots+s_{k} \leq s$, then $U_{s_{1}} \cdots U_{s_{k}} \subset U_{s}$. To see this, let $\left\{V_{s}\right\}$, $s=2^{-n}, n=0,1, \ldots$, be a family of neighborhoods of $G$ such that $\bigcap V_{s}=\{e\}$ and $V_{1}=G$. We will construct the family $\left\{U_{s}\right\}$ by induction in $n$. Let $U_{1}=V_{1}=G$. Then $\left\{U_{1}\right\}$ clearly has all the desired properties except M2. Now suppose we have constructed a family $\left\{U_{s}\right\}$, with $s=1, \ldots, 2^{-n}$ having all the desired properties except M2, and such that $U_{s} \subset V_{s}$. Choose a symmetric $U_{s / 2} \subset V_{s / 2}$ such that $\left(U_{s / 2}\right)^{2} \subset U_{s}$. Consider a sum $s_{1}+\cdots+s_{j}+2^{-n-1}+\cdots+2^{-n-1} \leq s_{0}$, where $s_{i} \in\left\{1, \ldots, 2^{-n}\right\}$ for $i \in 0,1, \ldots, j$ and there are $m$ terms $2^{-n-1}$. (We can assume $s_{0} \neq 2^{-n-1}$ because in that case the sum has one term and the argument is trivial.) Note that if $m^{\prime}$ is the smallest even integer $\geq m$, then $s_{1}+\cdots+s_{j}+m^{\prime} 2^{-n-1} \leq s_{0}$ (since $s_{0} \geq 2^{-n-1}$ ). We now have that

$$
\begin{array}{r}
U_{s_{1}} \cdots U_{s_{j}} \cdot\left(U_{2^{-n-1}}\right)^{m} \subset U_{s_{1}} \cdots U_{s_{j}} \cdot\left(U_{2^{-n-1}}\right)^{m^{\prime}} \\
\quad \subset U_{s_{1}} \cdots U_{s_{j}} \cdot\left(U_{2^{-n}}\right)^{m^{\prime} / 2} \subset U_{s_{0}},
\end{array}
$$


by the induction hypothesis. Since every $U_{s} \subset V_{s}$, M2 holds for the entire collection. To complete the construction of the family $\left\{U_{s}\right\}$ for $s \in \mathrm{R}^{+}$, let $U_{s}=\bigcup U_{s_{1}} \cdots U_{s_{k}}$, where the union is over all products $U_{s_{1}} \cdots U_{s_{k}}$ such that $s_{j} \in\{1,1 / 2, \ldots\}$ and $s_{1}+\cdots+s_{k} \leq s$. If we now let $U_{s}=U_{1}=G$ for all $s>1$, it is now straightforward to verify M5.

Definition 3.1. Let $G$ be a group. A family $\left\{U_{s}\right\}$ of sets satisfying conditions M1-M5 is called a metric family. The metric obtained from the formula 3.1 will be referred to as the associated metric. Given an invariant metric $d$ on $G$, the family $\left\{U_{s}\right\}$ of metric balls will be referred to as the metric family of $d$.

Recall that a space $(X, d)$ is called an inner metric space if for all $x, y \in X$, $d(x, y)=\inf \{l(c)\}$, where $c$ is a curve in $X$ joining $x$ and $y$, and $l(c)$ is the length of $c$. Inner metrics are the basis for most of metric geometry, including the geometry of Riemannian manifolds. If $d$ is a complete metric, then $d$ is an inner metric if and only if for each $x, y \in X$ and $\epsilon>0$ there exists $z \in X$ such that $d(x, z)+d(z, y)-$ $d(x, y) \leq \epsilon$, and $|d(x, z)-d(x, y) / 2|<\epsilon$. More strongly, if we can always find a midpoint $m$ between $x$ and $y$ (that is, $d(x, m)=d(y, m)=\frac{1}{2} d(x, y)$ ), then any pair of points can be joined by a minimal curve in $X$ (i.e., a curve whose length actually realizes the distance).

Proposition 3.1. Let $G$ be a group with complete invariant metric $d$. Then $d$ is an inner metric if and only if the associated family $\left\{U_{s}\right\}$ of $d$ is a geometry over $R^{+}$. If the geometry $\left\{U_{s}\right\}$ is strong, then every pair of points in $G$ can be joined by a minimal curve in $G$.

Proof. Suppose $d$ is an inner metric; then we need only show that $U_{s+t} \subset U_{s} U_{t}$. Suppose $x \in U_{s+t}$; suppose $s+t-d(e, x)=\epsilon>0$. Let $c$ be a curve from $e$ to $x$ of length $L<d(e, x)+\epsilon / 2$, and $y$ be on $c$ such that $d(e, y)=t-\epsilon / 2$. Then $d(y, x)+d(e, y) \leq L<d(e, x)+\epsilon / 2=s+t-\epsilon / 2$, so $d\left(e, y^{-1} x\right)=d(y, x)<s$; that is, $y^{-1} x \in U_{s}, y \in U_{t}$, and $y y^{-1} x=x$.

To prove the converse, given $\epsilon>0$ and $x \in G$ and letting $s=d(e, x)$, we need to find $y$ such that $d(e, y)+d(y, x)-s \leq \epsilon$, and $|d(e, y)-s / 2|<\epsilon$. Note that $x \in U_{s+\epsilon}=\left(U_{s / 2+\epsilon / 2}\right)^{2}$. That is, there exist $y, z \in U_{s / 2+\epsilon / 2}$ such that $y z=x$. But then $d(e, y)+d(y, x)<s / 2+\epsilon / 2+d(e, z)<s+\epsilon$. We also have $d(e, y)<s / 2+\epsilon / 2$ and $d(e, y) \geq d(e, x)-d(e, z) \geq s-s / 2-\epsilon / 2=s / 2-\epsilon / 2$.

For the last statement, note that if the geometry is strong, then modifying the above argument allows us to always find a midpoint between $e$ and $x$.

Note that in the above proof, completeness is only used in the second half (see the comments preceding Proposition 3.1).

Definition 3.2. Let $G$ be a group and $\left\{U_{s}\right\}$ be a metric (resp. inner metric) family on $G$, and let $H$ be a closed, normal subgroup of $G$. If $\pi: G \rightarrow G / H$ is the quotient map, the set $\left\{V_{s}\right\}$, where $V_{s}=\pi\left(U_{s}\right)$ is called the quotient metric (resp. inner metric) family on the group $G / H$.

It is trivial to verify that the quotient metric (resp. inner metric) family indeed satisfies M1-M5 (resp. G1-G5).

Proof of Proposition 1.6. For any $s>0, x \in G$,

$$
\begin{gathered}
\pi(B(x, s))=\pi\left(x U_{s}\right)=\pi\left(\left\{x y: y \in U_{s}\right\}\right)=\left\{\pi(x y): y \in U_{s}\right\}=\left\{\pi(x) \pi(y): y \in U_{s}\right\} \\
=\left\{\pi(x) z: z \in V_{s}\right\}=\pi(x) V_{s}=B(\pi(x), s) .
\end{gathered}
$$


Proof of Theorem 1.8. We first claim that $\pi^{\prime}$ is surjective. Let $x \in G=\pi(a)$, where $a=\left(x_{1}, \ldots, y_{1}, \ldots\right)$, with $x_{1} \in L, x_{i} \in G_{i-1}(i \geq 2)$ and $\left(y_{1}, \ldots\right) \in \mathrm{R}^{\omega}$. We will use the usual product notation in the factors $L \times G_{1} \times \cdots$, and addition notation in $\mathrm{R}^{\omega}$. Let $y_{i}^{\prime}=y_{i}-2^{-i}\left[2^{i} y_{i}\right]$, where $[y]$ is the greatest integer $\leq y$ (e.g. if $y_{i}>0$, then $y_{i}^{\prime}$ is the "remainder" beyond the largest multiple of $2^{-i} \leq y_{i}$ ). Then $0 \leq y_{i}^{\prime} \leq 2^{-i}$. Since the projection of $\operatorname{ker} \pi$ onto $\mathrm{R}^{\omega}$ is $\mathrm{Z} \times \frac{1}{2} \mathrm{Z} \times \cdots$, we can find $w \in \operatorname{ker} \pi$ of the form $\left(w_{1}, \ldots, y_{i}-y_{i}^{\prime}\right)$. But the element $a^{\prime}=\left(x_{1} w_{1}^{-1}, \ldots y_{1}^{\prime}, \ldots\right) \in L \times G_{1} \times \cdots \times E$ has the property that $a^{\prime-1} a=\left(w_{1}, \ldots, y_{i}-y_{i}^{\prime}\right) \in \operatorname{ker} \pi$, so $\pi\left(a^{\prime}\right)=\pi(a)=x$.

Provide $L$ with a left invariant Riemannian metric, each $G_{i}$ with a bi-invariant Riemannian metric so that the $\sum \delta\left(G_{i}\right)^{2}<\infty$ (where $\delta\left(G_{i}\right)$ denotes the diameter of $G_{i}$ ), and $E$ with the usual Hilbert space metric. Then the product metric on $\widetilde{G}_{M}:=L \times G_{1} \times \cdots \times E$ is an inner metric of curvature $\geq \min \{0, k\}$, where $k$ is a lower sectional curvature bound for $L$ (cf. [23]). (The product metric on a countable product $\prod\left(X_{i}, d_{i}\right)$ of metric spaces having square summable diameters is given by $d\left(\left(x_{1}, \ldots\right),\left(y_{1}, \ldots\right)\right)=\sqrt{\sum d_{i}\left(x_{i}, y_{i}\right)^{2}}$.) We claim that the inclusion $\widetilde{G}_{M} \hookrightarrow \widetilde{G}$ is continuous with respect to the metric topology on $\widetilde{G}_{M}$ and the product topology on $\widetilde{G}$. Let

$$
U=U_{1} \times \cdots \times U_{n} \times G_{n+1} \times \cdots \times I_{1} \times \cdots \times I_{m} \times \mathrm{R} \times \cdots
$$

be a basis element at $e$ in the topology of $\widetilde{G}$. Let $\epsilon>0$ be small enough that if $B_{i}=B_{i}(e, \epsilon)$ denotes the metric ball in $G_{i}$ centered at $e$ of radius $\epsilon$, then $B_{i} \subset U_{i}$ for all $1 \leq i \leq n$ and $(-\epsilon, \epsilon) \subset I_{j}$ for all $1 \leq j \leq m$. Since the projection onto any factor is distance non-increasing (with the product metric), we see that the metric ball $B=B(e, \epsilon)$ in $\widetilde{G}_{M}$ satisfies

$$
B \subset B_{1} \times \cdots \times B_{n} \times G_{n+1} \times \cdots \times(-\epsilon, \epsilon) \times \cdots \times(-\epsilon, \epsilon) \times \mathrm{R} \times \cdots \subset U .
$$

Since the inclusion $\widetilde{G}_{M} \hookrightarrow \widetilde{G}$ is continuous, we see that $\operatorname{ker} \pi^{\prime}=\operatorname{ker} \pi \cap \widetilde{G}_{M}$ is closed in $\widetilde{G}_{M}$. Note also that, as pointed out in the proof of Theorem 2.9 , $\operatorname{ker} \pi$ is central in $\widetilde{G}$, so ker $\pi^{\prime}$ is central, and hence normal, in $\widetilde{G}_{M}$.

Let $G^{\prime}:=\widetilde{G}_{M} / \operatorname{ker} \pi^{\prime}$ have the quotient metric (see Proposition 1.6). To conclude the proof we need to show that $G^{\prime}$ is topologically isomorphic to $G$. Consider the natural homomorphism $\phi: G^{\prime} \rightarrow G$ given by $\phi\left(g \operatorname{ker} \pi^{\prime}\right)=g \operatorname{ker} \pi$. We have already seen that $\phi$ is surjective, and clearly $\phi$ is also injective. A set in $G$ (resp. $G^{\prime}$ ) is open if and only if its pre-image in $\widetilde{G}$ (resp. $\widetilde{G}_{M}$ ) is open. Thus it is immediate from the continuity of the inclusion $\widetilde{G}_{M} \hookrightarrow \widetilde{G}$ that $\phi$ is continuous. Now consider $B(e, \epsilon) \subset G^{\prime}$. Choose $n$ large enough that $\sum_{i=n+1}^{\infty} \delta\left(G_{i}\right)^{2}<\epsilon^{2} / 3$ and $m$ large enough that $2^{-m}<\epsilon^{2} / 3$. Now let $\eta=\frac{\epsilon}{\sqrt{3(m+n)}}$ and consider the basis element

$$
V=B_{1}(e, \eta) \times \cdots \times B_{n}(e, \eta) \times G_{n+1} \times \cdots \times(-\eta, \eta) \times \cdots \times(-\eta, \eta) \times \mathrm{R} \times \cdots
$$

where there are $m$ copies of $(-\eta, \eta)$. Then for any $x \in \pi(V)$,

$$
d(x, e)^{2}<n \eta^{2}+\sum_{i=n+1}^{\infty} \delta\left(G_{i}\right)^{2}+m \eta^{2}+\sum_{i=m+1}^{\infty} 2^{-i}<\epsilon^{2},
$$

so $\pi(V) \subset B(e, \epsilon)$. This proves that $\phi$ is open, and completes the proof of the theorem. 
Restricting to $\widetilde{G}_{M}$ is necessary to induce any kind of invariant inner metric R on $G$, as we will now show. The proof of the next lemma is trivial (the geometry forms the desired basis). Still, this formulation seems to be useful. Note that any locally compact group satisfies the conclusion of the lemma.

Lemma 3.2. Let $G$ be a group with having a geometry $\left\{U_{r}\right\}$ over $\mathrm{R}^{+}$. Then there exists a basis $\mathcal{B}$ for the topology of $G$ at the identity such that for every $U, V \in \mathcal{B}$ there exists an $n$ such that $U^{n} \supset V$.

Proposition 3.3. The group $\mathrm{R}^{\infty}$ does not admit an invariant inner metric.

Proof. Suppose, to the contrary, that $\mathcal{B}$ is a basis satisfying the conclusion of Lemma 3.2. Any open set in $\mathrm{R}^{\infty}$ (hence any $U \in \mathcal{B}$ ) contains the set $C^{m}=$ $\left\{\left(0, \ldots, 0, x_{m+1}, x_{m+2}, \ldots\right): x_{k} \in \mathrm{R}, k>m\right\}$ for large enough $m$. Let $I$ be a bounded open interval in $\mathrm{R}$. Then the open set $V:=I^{m+1} \times \mathrm{R} \times \cdots$ has the property that $V^{n}$ does not contain $C^{m}$, and hence $U$, for any $n$. The same holds for any element of $\mathcal{B}$ contained contained in $V$, a contradiction.

Note that $\mathrm{R}^{\infty}$ is arcwise and locally arcwise connected, and metrizable. We can ask: does every group satisfying the previous three conditions and the conclusion to Lemma 3.2 admit a geometry over $\mathrm{R}^{+}$?

Proposition 3.4. Let $X, Y$ be (not necessarily inner) metric spaces and suppose $\phi: X \rightarrow Y$ is a weak submetry. If $X$ has curvature $\geq k$ for some $k$, then $Y$ has curvature $\geq k$.

Proof. First observe that $\phi$ has the following two properties: (1) it is distance nonincreasing and (2) for any points $x, y \in Y$ and $x^{\prime} \in X$ such that $\phi\left(x^{\prime}\right)=x$, there is a point $y^{\prime} \in X$ such that $\phi\left(y^{\prime}\right)=y$ and $d\left(x^{\prime}, y^{\prime}\right)$ is arbitrarily close to $d(x, y)$. Let $q \in Y$ be arbitrary. Since $X$ has curvature $\geq k$, then by definition ([2]) for any $p \in$ $X$, in particular for $p \in \phi^{-1}(q)$, there exists an open set $U$ such that if $a, b, c, d \in U$, $\alpha_{k}(a ; b, c)+\alpha_{k}(a ; b, d)+\alpha_{k}(a ; c, d) \leq 2 \pi$, where $\alpha_{k}(x ; y, z)$ denotes the representative angle in $S_{k}^{2}$ of the triple $(x ; y, z)$. We can assume, without loss of generality, that $U$ is an open metric ball $B_{X}(p, r)$. Let $V=B_{Y}(q, r / 3)$. Suppose that $x, y, z, w \in V$. By the above two properties we can find first a point $x^{\prime} \in B_{X}(p, r / 3)$ such that $\phi\left(x^{\prime}\right)=x$, then points $y^{\prime}, z^{\prime}, w^{\prime} \in U$ such that $d\left(x^{\prime}, v^{\prime}\right)$ is arbitrarily close to $d(x, v)$ and $\phi\left(v^{\prime}\right)=v$, where $v=y, z, w$. Now $\alpha_{k}\left(x^{\prime} ; y^{\prime}, z^{\prime}\right)+\alpha_{k}\left(x^{\prime} ; y^{\prime}, w^{\prime}\right)+\alpha_{k}\left(x^{\prime} ; z^{\prime}, w^{\prime}\right) \leq$ $2 \pi$ and since $\phi$ is distance non-increasing, $d\left(y^{\prime}, z^{\prime}\right) \geq d(y, z), d\left(y^{\prime}, w^{\prime}\right) \geq d(y, w)$, and $d\left(z^{\prime}, w^{\prime}\right) \geq d(z, w)$. Recall that the function $\alpha_{k}(x ; y, z)$, is monotone increasing as a function of $d(y, z)$ (fixing the other two distances), and is continuous in $x, y, z$, and $k$. It now follows that $\alpha_{k}(x ; y, z)+\alpha_{k}(x ; y, w)+\alpha_{k}(x ; z, w) \leq 2 \pi+\epsilon$ for any $\epsilon>0$.

Note that in the above proof it is clear that if $X$ has globally curvature $\geq k$ (i.e. $U=Y$ in the above notation), then $Y$ also has globally curvature $\geq k$.

\section{Geodesics and the fundamental group}

We first construct a simple example of an inner metric space and a curve having no geodesic in some (free or based) path homotopy class. Begin with the unit upper half circle $C$ in the plane; let $s(\theta)=(\cos \theta \pi, \sin \theta \pi)$. Join $s(0)$ and $s(1)$ by a segment in the plane. Now join $s(0)$ and $s(1 / 2)$ by a segment, and $s(1 / 2)$ and $s(1)$ by a segment. Next join $s(0)$ to $s(1 / 4), s(1 / 4)$ to $s(1 / 2), s(1 / 2)$ to $s(3 / 4)$, and 
so on. In the end we have constucted a set $S$ by adding infinitely many segments to $C$, and between any two points on $C$ there are two points joined by a segment. We take $S$ with the induced inner metric (that is, the distance between any two points is the length of the shortest path joining them). Clearly $C$ is not a geodesic; a shorter path between any two points on $C$ can always be found by moving along a segment instead. On the other hand, shortening $C$ always involves changing the homotopy class; hence $C$ has no shorter curve in its homotopy class. Note that this example is compact and finite dimensional but does not have a lower curvature bound (geodesics bifurcate).

We now show that similar behavior occurs in $T^{\infty}=\prod S_{i}$ with the product metric, where the $S_{i}$ have square summable diameters. Let $\gamma_{i}$ be one of the two unit parameterized geodesics in $S_{i}$ such that $\gamma_{i}(0)=e$, and define $\gamma(t)=$ $\left(\gamma_{1}(t), \gamma_{2}(2 t), \ldots\right) \in T^{\infty}$. Note that $\gamma$ is a 1 -parameter subgroup whose image in $T^{\infty}$ is a topologically a circle, but $\gamma$ is not a geodesic. To see this, note that for any $t$, if $2^{-i}<t$, then $\gamma_{i}:[0, t] \rightarrow S_{i}$ is a curve whose length exceeds the diameter of $S_{i}$. We can therefore find a shorter minimal curve $\alpha_{i}:[0, t] \rightarrow S_{i}$ joining $\gamma_{i}(0)$ and $\gamma_{i}(t)$, which extends to a geodesic also denoted by $\alpha_{i}$. But then $\gamma^{\prime}(t)=\left(\gamma_{1}(t), \ldots, \gamma_{i-1}\left(2^{i-1} t\right), \alpha_{i}(t), \gamma_{i+1}\left(2^{i+1} t\right), \ldots\right)$ is shorter than $\gamma$ between $\gamma(0)$ and $\gamma(t)$. From [23] we know that any unit geodesic $\beta$ in $T^{\infty}$ is of the form $\left(\beta_{1}\left(k_{1} t\right), \beta_{2}\left(k_{2} t\right), \ldots\right)$, where each $\beta_{i}$ is a unit geodesic in $S_{i}, \sum k_{i}=1$, and $\frac{\operatorname{diam} S_{i}}{k_{i}}>\delta$ for some fixed $\delta>0$. If $\beta$ is a geodesic loop and $\pi_{i}: T^{\infty} \rightarrow S_{i}$ is the projection, then $\beta_{i}=\pi_{i}(\beta)$ is a loop in $S_{i}$, so, letting $p_{i}$ denote $\pi_{i}$ restricted to $\beta$, we can consider $p_{i}$ as a mapping from $S^{1}$ onto $S^{1}$, whose degree is easily seen to be $\frac{k_{i}}{\operatorname{diam} S_{i}}$, which is bounded above by $\frac{1}{\delta}$. In other words, the components of a geodesic loop have bounded degree. A similar argument shows that the degree of $q_{i}=\left.\pi_{i}\right|_{\gamma}$ tends to infinity as $i$ becomes large. If $H$ were a homotopy between $\gamma$ and $\beta$, then $\pi_{i} \circ H$ would give rise to a homotopy between $p_{i}$ and $q_{i}$, which is a contradiction for large $i$. In a similar way, $\gamma$ is not null-homotopic; i.e., $\gamma$ represents a non-trivial homotopy class which contains no geodesic.

We make the following additional observations, whose proofs can be found in [27].

1. Every path homotopy class based at $e$ in $T^{\infty}$ contains a unique 1-parameter subgroup.

2. There are path homotopy classes containing no rectifiable curves.

3. The unique 1-parameter subgroup in a homotopy class in $T^{\infty}$ is the shortest path in the class if the class contains any rectifiable curve.

4. Even if it is rectifiable, the unique 1-parameter subgroup in any homotopy class $T^{\infty}$ may not be a geodesic. In this case there are rectifiable (and hence shortest) curves in the class, but no geodesics.

5. In general, even for compact Lie groups (e.g. $\mathrm{SO}(3)$ ), there may not be a unique 1-parameter subgroup in a given path homotopy class.

Note that in the universal covering group $\widetilde{G}$ (Theorem 1.5) of a compact, metrizable, arcwise connected group $G$, the factor $L$ is trivial. Since each compact Lie group has the property that every point lies on a 1-parameter subgroup, the same is true of $\widetilde{G}$, and so every homotopy class based at $e$ in $G$ contains a (possibly nonrectifiable in a given metric) 1-parameter subgroup. This fact was also shown earlier by Rickert in [25]. Such a statement is false even for non-compact Lie groups, but 
given a left-invariant Riemannian metric we can find (by passing to the universal cover) a geodesic in any homotopy class.

Proof of Theorem 1.10. Let $\widetilde{G}=L \times G_{1} \times \cdots \times \mathrm{R}^{\omega}$ be the universal covering group of $G$ and $\pi$ be the projection map. Let $\kappa$ represent some homotopy class based at $e$. Lift $\kappa$ to a curve $\widetilde{\kappa}$ in $\widetilde{G}$ based at $e$ ending at a point $a$. We can join $e$ to $a$ by a curve $\widetilde{c}$ which is a product $\widetilde{c}(t)=(\widetilde{\gamma}(t), \widetilde{\omega}(t))=(\widetilde{\gamma}(t), e)(e, \widetilde{\omega}(t))=(e, \widetilde{\omega}(t))(\widetilde{\gamma}(t), e)$ where $\widetilde{\gamma}$ is a geodesic in the factor $L$ (with the given Riemannian metric from Theorem 1.8) and $\widetilde{\omega}$ is a 1-parameter subgroup in the factor $G_{1} \times \cdots \times \mathrm{R}^{\omega}$. Now $c(t):=\pi(\widetilde{c}(t))=\gamma(t) \omega(t)=\omega(t) \gamma(t)$, where $\gamma(t)=\pi(\widetilde{\gamma}(t), e)$ and $\omega(t)=\pi(e, \widetilde{\omega}(t))$, and $c(t)$ lies in the same homotopy class as $\kappa$. Since $\omega(t)$ is trivially a 1-parameter subgroup, we need only show that $\gamma(t)$ is a geodesic. We can consider $\bar{\gamma}=(\widetilde{\gamma}, e)$ as a curve in $\widetilde{G}_{M}=L \times G_{1} \times \cdots \times l^{2}$. Note that $\bar{\gamma}$ is a geodesic in $\widetilde{G}_{M}$ with any other choice of metric on $G_{1} \times \cdots \times l^{2}$, and $\pi^{\prime}(\bar{\gamma})=\pi((\widetilde{\gamma}, e))=\gamma$. We now factor the mapping $\pi^{\prime}$ in the following way. Recall that $\widetilde{G}$ was constructed by first covering $G$ (in the usual sense - with discrete kernel) by the product group $L \times K$, where $K$ is a compact group, then by covering $K$ with the product $G_{1} \times \cdots \times \mathrm{R}^{\omega}$. Thus we have $\pi^{\prime}=\pi_{2} \circ \pi_{1}$, where $\pi_{2}: L \times K \rightarrow G$ has discrete kernel and $\pi_{1}: \widetilde{G}_{M} \rightarrow L \times K$ is of the form $I \times \pi^{\prime \prime}$, where $I: L \rightarrow L$ is the identity map and $\pi^{\prime \prime}: G_{1} \times \cdots \times l^{2}$ is the restriction of the covering map for $K$. We put bi-invariant Riemannian metrics on the $G_{i}$ having square summable diameters and a Euclidean metric on $l^{2}$, and take the product metric on $\widetilde{G}_{M}$. Let $L \times K$ have the quotient geometry induced by $\pi_{1}$. We claim that $\beta:=\pi_{1}(\bar{\gamma})$ is a geodesic in $L \times K$. Since the metric is invariant, we need only check that $\beta$ has a minimal segment about $e=\beta(0)$. Suppose that no segment $\beta_{t}$ of $\beta$ from $\beta(-t)$ to $\beta(t)$ is minimal. Let $l_{t}(\beta)$ denote the length of $\beta_{t}$. Then for all $t, d(\beta(-t), \beta(t))<l_{t}(\beta) \leq 2 t$ (the last inequality is because $\pi_{1}$ is distance non-increasing). Because $\pi_{1}$ is a weak submetry we can find a point $x_{t} \in G \times H$ such that $d\left(x_{t}, \bar{\gamma}(-t)\right)<2 t$ and $\pi\left(x_{t}\right)=\beta(t)$. However, $\operatorname{ker} \pi_{1}$ is contained in the slice $e \times G_{1} \times \cdots \times l^{2}$, so the projection $y_{t}$ of $x_{t}$ onto $L$ must be $\bar{\gamma}(t)$. For small enough $t$,

$$
2 t>d\left(x_{t}, \bar{\gamma}(-t)\right) \geq d\left(y_{t}, \bar{\gamma}(-t)\right)=d(\bar{\gamma}(-t), \bar{\gamma}(t))=2 t,
$$

a contradiction. (Here the second inequality is a property of the product metric and the second equality is because $\bar{\gamma}$ is a geodesic).

To finish the proof of the theorem, note that the quotient geometry on $G$ induced from $L \times K$ by $\pi_{2}$ is the same as the original geometry on $G$. Since ker $\pi_{2}$ is discrete, $\pi_{2}$ is a local isometry, and $\gamma=\pi_{2}(\beta)$ is a geodesic.

\section{ACKNOWLEDGEMENT}

We would like to thank the referee for his prompt and careful reading of this paper, and for providing us with some useful suggestions and Example 2.1.

\section{REFERENCES}

1. Anderson, R.D., and Bing, R.H., A complete elementary proof that Hilbert space is homeomorphic to the countably infinite product of lines, Bull of the AMS 74 (1968) 771-792. MR 37:5847

2. Berestovskii, V.N., Spaces with bounded curvature and distance geometry, Siberian Math. J. 27 (1986) 8-19. MR 88e:53110 (Russian original)

3. Berestovskii, V. N., Homogeneous spaces with intrinsic metric, Soviet Math. Dokl. 27 (1989) 60-63. 
4. Berestovskii, V. N., The structure of locally compact spaces with an intrinsic metric, Siberian Math. J. 30 (1989) 23-34. MR 90c:53120

5. Berestovskii, V. N., On Alexandrov's spaces with curvature bounded from above, Dokl. Rus. Akad. Nauk 324 (1995) 304-306.

6. Berestovskii, V. N. and Plaut, C., Homogeneous spaces of curvature bounded below, to appear, J. Geometric Analysis.

7. Bourbaki, N., Elements of Mathematics, General topology, Hermann, Paris, 1966. MR 34:5044b

8. Bousfield, A. and Kan, D., Homotopy limits, completions and localizations, Lecture Notes in Math., no. 304, Springer, Berlin, 1972. MR 51:1825

9. Brown, R., Higgins, P., and Morris, S., Countable products and sums of lines and circles: their closed subgroups, quotients and duality properties, Math. Proc. Camb. Phil. Soc. 78 (1975) 19-32. MR 56:12168

10. Cassels, J., and Fröhlich, A., Algebraic number theory, Thompson Book Company, Washington, D.C., 1967. MR 35:6500

11. Chevalley, C., Theory of Lie Groups, Princeton Univeristy Press, Princeton, N.J., 1946. MR 18:583c

12. Glushkov, V. M., Lie algebras of locally (bi)compact, Usp. Mat. Nauk 12 (1957) 137-142. MR 21:699

13. Glushkov, V.M., On the structure of connected locally (bi)compact groups, Mat. Sbornik (N.S.) 48(90) (1959) 75-91. MR 23:A957

14. Glushkov, V.M., The structure of locally compact groups and Hilbert's fifth problem, AMS Translations 15 (1960) 55-93. MR 22:5690

15. Gromov, M., Lafontaine, F., and Pansu, P., Structures métriques pour les variétés riemannienes, Cedic, Paris, 1981. MR 85e:53051

16. Helgason, S. Differential geometry, Lie groups, and symmetric spaces, Academic Press, New York, 1978. MR 80k:53081

17. Husemoller, D., Fibre bundles, McGraw-Hill, New York, 1966. MR 37:4821

18. Jacobson, N., Basic algebra II, Freeman, San Francisco, 1980. MR 81g:00001

19. Iwasawa, K. On some types of topological groups, Ann. of Math. 50 (1949) 507-558. MR 10:679a

20. Lashof, R., Lie algebras of locally compact groups, Pacific J. Math 7 (1957) 1145-1162. MR 19:1064a

21. Montgomery, D. and Zippin, L., Topological transformation groups, Interscience, New York, 1955. MR 17:383b

22. Munkres, J., Topology: a first course, Prentice-Hall, New Jersey, 1975. MR 57:4063

23. Plaut, C., Geometrizing infinite-dimensional locally compact groups, Trans. of the AMS, 348 (1996) 941-962. MR 96h:53088

24. Pontryagin, L. S., Topological groups, Gordon and Breach, New York, 1966. MR 34:1439

25. Rickert, N., Arcs in locally compact groups, Math. Annalen 172 (1967) 222-228. MR 35:4331

26. Spanier, E., Algebraic Topology, Springer, Berlin, 1966. MR 35:1007

27. Stallman, C., Dissertation, University of Tennessee, 1996.

Department of Mathematics, Omsk State University, Pr. Mira 55A, Omsk 77644077 Russia

E-mail address: berest@univer.omsk.su

Department of Mathematics, University of Tennessee, Knoxville, Tennessee 37996

E-mail address: plaut@novell.math.utk.edu

Department of Mathematics and Computer Science, Augusta State University, AuGusta, Georgia 30904-2200 